\title{
On solving uncooperative linear bilevel multi-follower programming problems
}

\author{
Fatemeh Moslemi ${ }^{1}$, Habibe Sadeghi $^{2 *}$ \\ ${ }^{*}$ Corresponding author
}

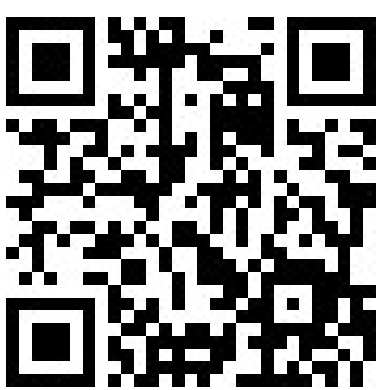

1. Department of Mathematics, Faculty of Mathematical Sciences and Computer, Shahid Chamran University of Ahvaz, Ahvaz, Iran, moslemi.fatemeh@gmail.com

2. Department of Mathematics, Faculty of Mathematical Sciences and Computer, Shahid Chamran University of Ahvaz, Ahvaz, Iran, habibe.sadeghi@scu.ac.ir

\begin{abstract}
The relationship between the reference-uncooperative linear bilevel two-follower decision making and the multiobjective decision making has been recently considered (Sadeghi and Moslemi, 2019). In this paper, we address the foregoing relation for the uncooperative linear bilevel multi-follower programming (ULBMFP) model with $k \geq 2$ followers. Furthermore, we consider some geometric properties of the feasible solutions set of the ULBMFP problem. Moreover, an algorithm to find an optimal solution for the ULBMFP problem was proposed. Ultimately, some numerical examples to illustrate the proposed algorithm were provided.
\end{abstract}

Key Words: Bilevel programming; Efficient set; Multi-follower programming; Multi-objective programming.

Mathematical Subject Classification: 90C29, 90C08.

\section{Introduction}

Multilevel programming (MLP) problem is developed to deal with the decentralized decision-making situations in which decision-makers are arranged within a hierarchical structure. Bilevel programming (BLP) problem is a special case of the MLP problem when only two levels exist. Most of the research concerning the MLP problem have focused on the BLP problem (Bard, 1998; Dempe, 2003). The BLP problem has many applications in different domains, such as those in supply chain management (Ma et al., 2014), planning (Miao et al., 2017), logistics (Safaei et al., 2018), and energy management (Alipour et al., 2018). That is the reason why so much attention has been paid to the BLP problems in recent years.

In the BLP problem, a decision-maker at the upper level is termed as a leader, and at the lower level is termed as a follower. The BLP problem may involve multiple decision-makers at the lower level, and these followers may have different reactions to a possible decision by the leader. In this case, the BLP problem is called a bilevel multi-follower programming (BLMFP) problem. Based on the relationship among followers, the BLMFP problems are classified into several classes. In this study, we consider a class of linear BLMFP problems wherein each follower controls a separate set of decision variables and attempts to optimize its own objective function over its own constraints. It is called an uncooperative linear bilevel multi-follower programming (ULBMFP) problem (Zhang et al., 2016). Several researchers have proposed solution approaches to solving the BLMFP problems. For example, Faísca et al. (2007), presented the multi-parametric programming approach to solving the linear BLMFP problems. Lu et al. (2007), presented an extended Kuhn-Tuker approach to solving the BLMFP problem in a referential-uncooperative situation. For a good bibliography, see Zhang et al. (2016). However, there exist very few papers on applying the multi-objective program- 
ming techniques to solving the BLP problems and the BLMFP ones due to their subjective complexity (Pieume et al., 2009; Wen and Hsu). As an example, in Glackin et al. (2009), two algorithms based on the multi-objective linear programming (MOLP) techniques to solve the linear bilevel programming (LBLP) problems were presented. The proposed algorithms were usable for LBLP problems containing only one follower while there exist multiple followers in most applications.

In this paper, a relationship between ULBMFP problems and MOLP problems is presented. This relationship is motivated by the relationship between LBLP problems and MOLP problems (Fülöp, 1993). Furthermore, we investigated some geometric properties of the feasible solutions set of the ULBMFP problem. Also, an algorithm to solve ULBMFP problems is presented.

The paper is organized as follows. In Section 2, we introduce some basic definitions and notations about ULBMFP problems and MOLP problems. In Section 3, we introduce $k$ MOLP problems such that each feasible extreme point for the ULBMFP problem, with $k$ followers, is an efficient extreme point for all of these $k$ MOLP problems. Next, we prove that the ULBMFP problem can be reduced to optimize the leader objective function over a certain efficient set. Moreover, we discuss some geometric properties of the feasible solutions set of the ULBMFP problem in special cases. Based on these results, Section 4 proposes an algorithm for solving the ULBMFP problem. Section 5 presents some numerical examples to illustrate the proposed algorithm. Finally, the paper is included in Section 6.

\section{Preliminaries}

First, we will introduce the formulation of the ULBMFP problem, then, we state the definition of the MOLP problem and some notations that are used in the rest of the paper.

\subsection{Linear bilevel multi-follower programming problem}

We consider a linear bilevel programming problem with $k(k \geq 2)$ followers. We assume that there are no shared decision variables, objective functions, and constraints among the followers. This is called an uncooperative linear bilevel multi-follower programming (ULBMFP) problem (Zhang et al., 2016). This model is defined as follows:

$$
\begin{aligned}
& \min _{x \in X} F\left(x, y_{1}, \ldots, y_{k}\right)=c^{T} x+\sum_{i=1}^{k} d_{i}^{T} y_{i}, \\
& \quad \text { s.t. } A x \leq b, \\
& \quad \min _{y_{i} \in Y_{i}} f_{i}\left(x, y_{i}\right)=e_{i}^{T} y_{i}, \quad i=1,2, \ldots, k, \\
& \quad \text { s.t. } A_{i} x+B_{i} y_{i} \leq b_{i},
\end{aligned}
$$

where $x \in X \subset \mathbb{R}^{n}, y_{i} \in Y_{i} \subset \mathbb{R}^{m_{i}}, F: X \times Y_{1} \times \ldots \times Y_{k} \rightarrow \mathbb{R}$, and $f_{i}: X \times Y_{i} \rightarrow \mathbb{R}, i=1,2, \ldots, k$. Also, $c \in \mathbb{R}^{n}, d_{i}, e_{i} \in \mathbb{R}^{m_{i}}, A \in \mathbb{R}^{p \times n}, b \in \mathbb{R}^{p}, A_{i} \in \mathbb{R}^{q_{i} \times n}, B_{i} \in \mathbb{R}^{q_{i} \times m_{i}}$, and $b_{i} \in \mathbb{R}^{q_{i}}, i=1,2, \ldots, k$. Although $x \geq 0$, and $y_{i} \geq 0$, for $i=1,2, \ldots, k$, do not explicitly appear in this problem, we assume that they exist in the set of constraints.

Notice that for each follower, the value of variable $x$ is given. Thus, a problem equivalent to the ULBMFP problem (1) is obtained if the followers' objective functions are replaced by $f_{i}\left(x, y_{i}\right)=c_{i}^{T} x+e_{i}^{T} y_{i}$, for $i=1,2, \ldots, k$, where $c_{i} \in \mathbb{R}^{n}$.

We introduce some definitions and notations as to the ULBMFP problem (1) as follows (Bard, 1998; Zhang et al., 2016):

(1) The constraint region of the ULBMFP problem:

$$
S=\left\{\left(x, y_{1}, \ldots, y_{k}\right) \in X \times Y_{1} \times \ldots \times Y_{k}: A x \leq b, A_{i} x+B_{i} y_{i} \leq b_{i}, i=1,2, \ldots, k\right\} .
$$

(2) The feasible region of the $i$ th follower for each $x \in\{x \in X: A x \leq b\}$ :

$$
S_{i}(x)=\left\{y_{i} \in Y_{i}: B_{i} y_{i} \leq b_{i}-A_{i} x\right\}, i=1,2, \ldots, k .
$$


Thus the feasible region of the $i$ th follower is affected by the leader's choice.

(3) The projection of $S$ onto the leader's decision space:

$$
S(X)=\left\{x \in X: \exists\left(y_{1}, \ldots, y_{k}\right) \in Y_{1} \times \ldots \times Y_{k},\left(x, y_{1}, \ldots, y_{k}\right) \in S\right\} .
$$

(4) The $i$ th follower's rational reaction set for $x \in S(X)$ :

$$
P_{i}(x)=\left\{y_{i} \in Y_{i}: y_{i} \in \operatorname{argmin}\left[f_{i}\left(x, \hat{y}_{i}\right): \hat{y}_{i} \in S_{i}(x)\right]\right\}, \quad i=1,2, \ldots, k,
$$

where

$$
\operatorname{argmin}\left[f_{i}\left(x, \hat{y}_{i}\right): \hat{y}_{i} \in S_{i}(x)\right]=\left\{y_{i} \in S_{i}(x): f_{i}\left(x, y_{i}\right) \leq f_{i}\left(x, \hat{y}_{i}\right), \forall \hat{y}_{i} \in S_{i}(x)\right\}
$$

The followers observe the leader's action and simultaneously react by selecting $y_{i}$ from their feasible set to minimize their objective function.

(5) The inducible region $(I R)$ of the problem (1):

$$
I R=\left\{\left(x, y_{1}, \ldots, y_{k}\right):\left(x, y_{1}, \ldots, y_{k}\right) \in S, y_{i} \in P_{i}(x), i=1,2, \ldots, k\right\} .
$$

The inducible region is the set of feasible solutions to the ULBMFP problem. Thus, determining a solution for the ULBMFP problem is equivalent to solving the following problem:

$$
\min \left\{F\left(x, y_{1}, \ldots, y_{k}\right):\left(x, y_{1}, \ldots, y_{k}\right) \in I R\right\}
$$

To ensure that the ULBMFP problem is well-posed as well as has an optimal solution (Zhang et al., 2016), we assumed that the following assumptions hold:

(1) $S$ is non-empty and compact.

(2) $I R$ is non-empty.

(3) $P_{i}(x) \neq \emptyset$ and it is a point-to-point map for all leader's choices of $x$, for $i=1,2, \ldots, k$.

Note that, at the above ULBMFP problem, both the objective function and the set of constraints of each follower only include the leader variables and one's own variables. This fact implies that the followers do not share any information. These kinds of followers are called independent followers by Calvate et al. (2007). In this paper, we used a similar trick in Glackin et al. (2009) to reformulate the ULBMFP problem as the MOLP problem.

\subsection{Multi-objective linear programming problem}

Assume that $m \geq 2$ is an integer and $c_{i} \in \mathbb{R}^{n}, i=1,2, \cdots, m$ are row vectors. Let $C$ be a $m \times n$ matrix whose $i$-th row is given by $c_{i}, i=1,2, \cdots, m$. Also, $U$ is a non-empty, compact and convex polyhedral set in $\mathbb{R}^{n}$. A multi-objective linear programming (MOLP) problem is formulated in general as follows:

$$
\min \{C X: X \in U\}
$$

where $U$ is called feasible region.

Definition 2.1. (Steure, 1986) A point $\bar{X} \in U$ is an efficient solution if there exists no $X \in U$ such that $C X \leqslant C \bar{X}$ and $C X \neq C \bar{X}$. Otherwise, $\bar{X}$ is an inefficient solution.

Let $E$ denote the set of all efficient solutions of the MOLP problem. Then, the set $E$ is non-empty (Ehrgott, 2005, Theorem 2.19).

Also, the following definitions are used in a sequel:

Definition 2.2. (Steure, 1986) Let $U \subseteq \mathbb{R}^{n}$, and $\bar{X}, \overline{\bar{X}} \in U$. The notation $\gamma(\bar{X}, \overline{\bar{X}})$ is the set of all strictly convex combinations of $\bar{X}$ and $\overline{\bar{X}}$, i.e.,

$$
\gamma(\bar{X}, \overline{\bar{X}})=\{\hat{X} \in U: \hat{X}=\alpha \bar{X}+(1-\alpha) \overline{\bar{X}}, \text { for some } \alpha, 0<\alpha<1\} .
$$


Similarly, $\gamma[\bar{X}, \overline{\bar{X}})$ and $\gamma(\bar{X}, \overline{\bar{X}}]$ are defined as follows:

$$
\begin{aligned}
& \gamma[\bar{X}, \overline{\bar{X}})=\{\hat{X} \in U: \hat{X}=\alpha \bar{X}+(1-\alpha) \overline{\bar{X}}, \text { for some } \alpha, 0<\alpha \leqslant 1\}, \\
& \gamma(\bar{X}, \overline{\bar{X}}]=\{\hat{X} \in U: \hat{X}=\alpha \bar{X}+(1-\alpha) \overline{\bar{X}}, \text { for some } \alpha, 0 \leqslant \alpha<1\} .
\end{aligned}
$$

\section{Main results}

In this section, we will introduce $k$ MOLP problems such a way that each efficient extreme solution for all of these $k$ problems simultaneously is a feasible extreme solution (in other words, an extreme point of $I R$ ) to the ULBMFP problem.

Note that $\bar{X}=\left(\bar{x}, \bar{y}_{1}, \ldots, \bar{y}_{k}\right) \in I R$, implies that $\bar{X}=\left(\bar{x}, \bar{y}_{1}, \ldots, \bar{y}_{k}\right) \in S$ and $\bar{y}_{i} \in P_{i}(\bar{x})$, for $i=1,2, \ldots, k$. Therefore, $\bar{y}_{i}$ is an optimal solution for the following linear programming (LP):

$$
\begin{aligned}
& \min _{y_{i}} f_{i}\left(\bar{x}, y_{i}\right)=e_{i}^{T} y_{i}, \\
& \text { s.t. } B_{i} y_{i} \leq b_{i}-A_{i} \bar{x} .
\end{aligned}
$$

Consider the ULBMFP problem (1). We assume that $r=n+\sum_{j=1}^{k} m_{j}, r_{i}=n+\sum_{\substack{j=1 \\ j \neq i}}^{k} m_{j}$, and $k_{i}=r_{i}+2, i=1,2, \ldots, k$.

Let the $k_{i} \times r$ criterion matrices $C_{i}, i=1,2, \ldots, k$ be defined as follows:

$$
C_{i}=\left[\begin{array}{cccccccc}
I_{n} & O & \ldots & O & O & O & \ldots & O \\
O & I_{m_{1}} & \ldots & O & O & O & \ldots & O \\
\vdots & \vdots & \ddots & \vdots & \vdots & \vdots & \ddots & \vdots \\
O & O & \ldots & I_{m_{i-1}} & O & O & \ldots & O \\
O & O & \ldots & O & O & I_{m_{i+1}} & \ldots & O \\
\vdots & \vdots & \ddots & \vdots & \vdots & \vdots & \ddots & \vdots \\
O & O & \ldots & O & O & O & \ldots & I_{m_{k}} \\
-e^{T} & -e^{T} & \ldots & -e^{T} & 0 & -e^{T} & \ldots & -e^{T} \\
0 & 0 & \ldots & 0 & e_{i}^{T} & 0 & \ldots & 0
\end{array}\right],
$$

where $I_{n}, I_{m_{i}}, i=1,2, \ldots, k$ are identity matrices, $O$ and 0 , are zero matrices and zero vectors of conformal dimension, respectively. Also, $e$ is a vector having each entry equal to 1 .

Now, we define the following k MOLP problems:

$$
\min \left\{C_{i} X: X \in S\right\}, \quad i=1,2, \ldots, k .
$$

Let $E_{i}, i=1,2, \ldots, k$ be the set of efficient solutions of the MOLP $i, i=1,2, \ldots, k$. The following proposition holds:

Lemma 3.1. Let $I R$ and $E_{i}, i=1,2, \ldots, k$ be defined as above. Then $I R \subseteq E_{i}$, for all $i=1,2, \ldots, k$.

Proof. Assume that $\bar{X}=\left(\bar{x}, \bar{y}_{1}, \ldots, \bar{y}_{k}\right) \in I R$. It suffices to show that $\bar{X}$ is an efficient solution to the MOLPi, for all $i=1,2, \ldots, k$. Let us suppose the contrary, i.e., there exists $X \in S$ and some $i \in\{1,2, \ldots, k\}$ such that $C_{i} X \leqslant C_{i} \bar{X}$ and $C_{i} X \neq C_{i} \bar{X}$. Because $C_{i} X \leqslant C_{i} \bar{X}$, using the structure of matrix $C_{i}$, one obtains:

$$
\begin{array}{r}
x \leq \bar{x}, y_{l} \leq \bar{y}_{l}, l=1,2, \ldots, k, l \neq i, \\
-e^{T} x-\sum_{\substack{l=1 \\
l \neq i}}^{k} e^{T} y_{l} \leqslant-e^{T} \bar{x}-\sum_{\substack{l=1 \\
l \neq i}}^{k} e^{T} \bar{y}_{l} .
\end{array}
$$


From the relation (5), one obtains:

$$
-e^{T} x-\sum_{\substack{l=1 \\ l \neq i}}^{k} e^{T} y_{l} \geq-e^{T} \bar{x}-\sum_{\substack{l=1 \\ l \neq i}}^{k} e^{T} \bar{y}_{l} .
$$

The last two relations imply that:

$$
-e^{T} x-\sum_{\substack{l=1 \\ l \neq i}}^{k} e^{T} y_{l}=-e^{T} \bar{x}-\sum_{\substack{l=1 \\ l \neq i}}^{k} e^{T} \bar{y}_{l} .
$$

Thus, from the inequality (5) and equality (6), we conclude that:

$$
x=\bar{x}, y_{l}=\bar{y}_{l}, l=1,2, \ldots, k, l \neq i .
$$

Obviously, $y_{i}$ is a feasible solution for the problem (3)-(4). Due to $C_{i} X \neq C_{i} \bar{X}$ and equalities (6) and (7), we conclude that $e_{i}^{T} y_{i}<e_{i}^{T} \bar{y}_{i}$ which contradicts $\bar{y}_{i}$ being an optimal solution for the problem (3)-(4). This completes the proof.

Now, assume that

$$
E=\bigcap_{i=1}^{k} E_{i}
$$

One can get from Proposition 3.1:

Remark 3.1. Let $I R$ and $E_{i}, i=1,2, \ldots, k$ be defined as above. Then, $I R \subseteq \bigcap_{i=1}^{k} E_{i}=E$, and regarding Assumption (2), E is a non-empty set.

Theorem 3.1. The extreme points of $I R$ and $E$ are the same.

Proof. Let $\bar{X}=\left(x, y_{1}, \ldots, y_{k}\right) \in I R_{e x}$ be arbitrary. Then, $\bar{X}$ is an extreme point of $S$ (Zhang et al., 2016, Corollary 4.3). Because $I R \subseteq E$, we get $\bar{X} \in E$. Therefore, $\bar{X}$ is an extreme point of $E$. Since $\bar{X} \in I R_{e x}$ was chosen arbitrarily, we get $I R_{e x} \subseteq E_{e x}$. Now, we will show that $E_{e x} \subseteq I R_{e x}$. Suppose that $\bar{X}=\left(x, y_{1}, \ldots, y_{k}\right) \in E_{e x}$. There are two cases, $\bar{X} \notin I R$ or $\bar{X} \in I R$ :

Case 1: $\bar{X} \notin I R$.

In this case, it follows from the definition of $I R$ that there is at least an index $i_{0}, 1 \leq i_{0} \leq k$ such that $y_{i_{0}} \notin P_{i_{0}}(x)$. By Assumption (3), $P_{i_{0}}(x)$ is non-empty and singleton. Then, there exists $\hat{y}_{i_{0}} \in S_{i_{0}}(x)$ such that $\hat{y}_{i_{0}} \in P_{i_{0}}(x)$ and so $f_{i_{0}}\left(x, \hat{y}_{i_{0}}\right)<f_{i_{0}}\left(x, y_{i_{0}}\right)$. Consequently, we get:

$$
e_{i_{0}}^{T} \hat{y}_{i_{0}}<e_{i_{0}}^{T} y_{i_{0}} .
$$

Set $\hat{X}=\left(x, y_{1}, \ldots, y_{i_{0}-1}, \hat{y}_{i_{0}}, y_{i_{0}+1}, \ldots, y_{k}\right)$. Now, we show $\hat{X} \in S$. Because $\bar{X} \in E_{e x} \subseteq S$, we have $A x \leq b$ and $y_{i} \in S_{i}(x)$ for all $i \neq i_{0}$. Furthermore, $\hat{y}_{i_{0}} \in S_{i_{0}}(x)$. Hence, $\hat{X} \in S$. Due to the structure of matrix $C_{i_{0}}$ and inequality (8), we have $C_{i_{0}} \hat{X} \leqslant C_{i_{0}} \bar{X}, C_{i_{0}} \hat{X} \neq C_{i_{0}} \bar{X}$, which contradicts $\bar{X} \in E \subseteq E_{i_{0}}$. Hence $\bar{X} \in I R$.

Case 2: $\bar{X} \in I R$.

Because $\bar{X} \in E_{e x}$, and $I R \subseteq E, \bar{X}$ is an extreme point of $I R$. Therefore, $E_{e x} \subseteq I R_{e x}$. This completes the proof.

Now, consider the following mathematical program:

$$
\min \left\{F\left(x, y_{1}, \ldots, y_{k}\right):\left(x, y_{1}, \ldots, y_{k}\right) \in E\right\} .
$$

It is known that the efficient set $E$ is closed. Also, since $E$ is a closed subset of the compact polyhedral set of $S$, itself is a compact set. Thus, problem (9) involves the optimization of a linear function over a compact set. Hence, there exists an optimal solution to the problem (9), i.e., $E^{*} \neq \emptyset$ (Bazaraa et al., 2006, Theorem 2.3.1). Therefore, there is 
an extreme point of $E$, which is an optimal solution for the problem (9) (Sadeghi and Moslemi, 2019, Corollary 1).

The relation between the ULBMFP problem and the problem (9) is as follows:

Theorem 3.2. A point $\bar{X}=\left(\bar{x}, \bar{y}_{1}, \ldots, \bar{y}_{k}\right) \in I R_{e x}$ is an optimal solution to the ULBMLP problem if and only if it is an optimal solution to the problem (9).

Proof. The proof is similar to that of Theorem 3 in Sadeghi and Moslemi (2019).

Also, similar to the reference-uncooprative linear bilevel multi-follower programming problem (Sadeghi and Moslemi, 2019), one can prove that $E \subseteq$ convIR for the ULBMLP problem where convIR is the smallest convex set containing $I R$. If the efficient set $E$ is a convex set, one can obtain $E=\operatorname{conv} I R$. Also, if the inducible region $I R$ is a convex set, one can obtain $E=I R$.

Now, we prove the following lemma:

Lemma 3.2. Let $\bar{X}$ and $\overline{\bar{X}}$ be distinct points in $S$ and $\bar{X} \notin I R$. Then, none of the points on the line segment $\gamma[\bar{X}, \overline{\bar{X}})$ belong to $I R$.

Proof. Let $\bar{X}=\left(\bar{x}, \bar{y}_{1}, \ldots, \bar{y}_{k}\right), \overline{\bar{X}}=\left(\overline{\bar{x}}, \overline{\bar{y}}_{1}, \ldots, \overline{\bar{y}}_{\underline{k}}\right)$, and $\tilde{X}=\left(\tilde{x}, \tilde{y}_{1}, \ldots, \tilde{y}_{k}\right) \in \gamma[\bar{X}, \overline{\bar{X}})$ be arbitrary. Then, there exists $0<\tilde{\alpha} \leqslant 1$ such that $\tilde{X}=\tilde{\alpha} \bar{X}+(1-\tilde{\alpha}) \overline{\bar{X}}$. Since $\bar{X} \notin I R$, there is at least an index $i_{0} \in\{1,2, \ldots, k\}$ such that $\bar{y}_{i_{0}} \notin P_{i_{0}}(\bar{x})$. It follows from Assumption (3) that there exists $\hat{y}_{i_{0}} \in S_{i_{0}}(\bar{x})$ such that $\hat{y}_{i_{0}} \in P_{i_{0}}(\bar{x})$. Thus, $f_{i_{0}}\left(\bar{x}, \hat{y}_{i_{0}}\right)<f_{i_{0}}\left(\bar{x}, \bar{y}_{i_{0}}\right)$. We set $\hat{X}=\tilde{\alpha}\left(\bar{x}, \bar{y}_{1}, \ldots, \bar{y}_{i_{0}-1}, \hat{y}_{i_{0}}, \bar{y}_{i_{0}+1}, \ldots, \bar{y}_{k}\right)+(1-\tilde{\alpha})\left(\overline{\bar{x}}, \overline{\bar{y}}_{1}, \ldots, \overline{\bar{y}}_{i_{0}-1}, \overline{\bar{y}}_{i_{0}}, \overline{\bar{y}}_{i_{0} \pm 1}, \ldots, \overline{\bar{y}}_{k}\right)$. Note that $\tilde{x}=\hat{x}$, and $\tilde{y}_{i}=\hat{y}_{i}$, for $i=1,2, \ldots, k, i \neq i_{0}$. Since $\left(\bar{x}, \bar{y}_{1}, \ldots, \bar{y}_{i_{0}-1}, \hat{y}_{i_{0}}, \bar{y}_{i_{0}+1}, \ldots, \bar{y}_{k}\right) \in S, \overline{\bar{X}} \in S$ and $S$ is a convex set, $\hat{X} \in S$. Consequently, we have $f_{i_{0}}(\hat{X})<f_{i_{0}}(\tilde{X})$, and so $\tilde{y}_{i_{0}} \notin P_{i_{0}}(\tilde{x})$. Hence $\tilde{X} \notin I R$. Since $\tilde{X} \in \gamma[\bar{X}, \overline{\bar{X}})$ was chosen arbitrarily, we conclude that $\gamma[\bar{X}, \overline{\bar{X}}) \notin I R$. This fact completes the proof.

We immediately get the following corollary to Lemma 3.2.

Corollary 3.1. Let $\bar{X}, \overline{\bar{X}} \in I R$, and $X \in \gamma(\bar{X}, \overline{\bar{X}})$ with $X \notin I R$. Then, none of the points on line segment $\gamma(\bar{X}, \overline{\bar{X}})$ belongs to $I R$.

Proof. According to Lemma 3.1, because $\overline{\bar{X}} \in I R$ and $X \notin I R$, then $\gamma[X, \overline{\bar{X}}) \notin I R$. Similarly, because $\bar{X} \in I R$ and $X \notin I R$, then $\gamma(\bar{X}, X] \notin I R$. Thus, $\gamma(\bar{X}, \overline{\bar{X}}) \notin I R$.

Now, consider the following definitions:

Definition 3.1. (Bazaraa et al., 1977) A path is a sequence of edges that joins a sequence of distinct extreme points $\bar{X}, \ldots, \hat{X}$.

Definition 3.2. (Bazaraa et al., 1977) A closed path is a path from some extreme point $\bar{X}$ to $\hat{X}$ plus the $\gamma(\hat{X}, \bar{X})$, i.e., the sequence of edges in which the initial and terminal extreme points of the path are the same.

By Remark 3.1, we have $I R \subseteq E$ whereas if $E$ and $I R$ are the connected union of edges without any closed path, this inclusion turns into equality. The following lemma proves this property:

Lemma 3.3. If the inducible region $I R$ and the efficient set $E$ are the connected union of edges without any closed path, then $E=I R$.

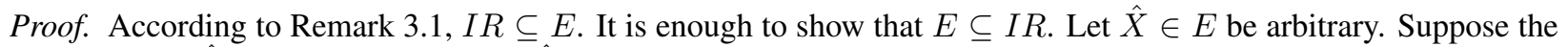
contrary, i.e., $\hat{X} \notin I R$. It is clear that $\hat{X}$ is not an extreme point of $E$, (Note that $I R_{e x}=E_{e x}$ ). Hence, there exist $\bar{X} \in E_{e x}$ and $\overline{\bar{X}} \in E_{e x}$ (and so belong to $I R_{e x}$ ) such that $\hat{X} \in \gamma(\bar{X}, \overline{\bar{X}})$. According to the Corollary 3.1, because $\hat{X} \notin I R$, then $\gamma(\bar{X}, \overline{\bar{X}}) \notin I R$. Since $I R$ is connected, there exists a path of edges between $\bar{X}$ and $\bar{X}$ in $I R$ and so in $E$. Since $\hat{X} \in E$ and $E$ is connected, we get $\gamma(\bar{X}, \overline{\bar{X}}) \in E$. Therefore, there exists a closed path in $E$, which contradicts the fact that $E$ does not contain a closed path. Thus, $E \subseteq I R$. This completes the proof. 


\section{The algorithm}

In this section, we present a modified version of the proposed algorithm in Sadeghi and Moslemi (2019) for the ULBMFP problem (1). The modified algorithm can be described as follows:

\section{The algorithm}

Step 1. Construct the MOLPi, and solve it to obtain the efficient set $E_{i}$, for all $i=1,2, \ldots, k$.

- One can use the presented approaches in Pieume et al. (2008); Sayin (1996); and Steure (1986) in Step 1.

Step 2. Set $E=\bigcap_{i=1}^{k} E_{i}$.

Step 3. Solve the following LP:

$$
\min \left\{F\left(x, y_{1}, \ldots, y_{k}\right):\left(x, y_{1}, \ldots, y_{k}\right) \in S\right\} .
$$

Let $X^{*}$ be an optimal solution. If $X^{*} \in E$, stop. Thus, $X^{*}$ is an optimal solution to the ULBMFP problem. Otherwise, go to Step 4.

- Note that one can use the known solution methods for LPs (Bazaraa et al., 1977), in Step 3.

Step 4. Find an optimal solution to the problem (9). Let $X^{*}$ be an optimal solution for the problem (9). Therefore, it is an optimal solution to the ULBMFP problem.

- For instance, the developed approaches in Benson (1984); Horst et al. (2007); Jorge (2005); and Metev (2007) can be used in Step 4.

Similar to the proposed algorithm in Sadeghi and Moslemi (2019), Step 3 of the algorithm checks if $X^{*} \in E$. If $X^{*} \in E$ then the algorithm stops at Step 3 and does not enter Step 4. Thus, Step 3 leads to a reduction of computations in some cases and it is not a redundancy. Also, we know that there exists an extreme point of $E$ which is an optimal solution for the problem (9). Hence, in Step 4, for a few variables, one can find an optimal solution by picking the minimum value of the objective function among all extreme points of $E$.

\section{Numerical examples}

In this section, we solve two numerical examples by the proposed algorithm.

Example 5.1. Consider the following ULBMFP problem.

$$
\begin{gathered}
\min _{x} F\left(x, y_{1}, y_{2}\right)=x+2 y_{1}-5 y_{2}, \\
\text { s.t. } x \geq 0 \\
\min _{y_{1}} f_{1}\left(x, y_{1}\right)=-y_{1}, \\
\text { s.t. } x+y_{1} \leq 3, \\
y_{1} \geq 0, x \geq 0 \\
\min _{y_{2}} f_{2}\left(x, y_{2}\right)=-y_{2}, \\
\text { s.t. } 2 x+y_{2} \leq 2, \\
0 \leq y_{2} \leq 1, x \geq 0
\end{gathered}
$$

In this problem, we have:

$$
S=\left\{\left(x, y_{1}, y_{2}\right): x \geq 0, x+y_{1} \leq 3, y_{1} \geq 0,2 x+y_{2} \leq 2,0 \leq y_{2} \leq 1\right\} .
$$


Also, one can see that the extreme points of $S$ are as follows:

$$
\begin{aligned}
& a_{1}=(0,0,0), a_{2}=(0,3,0), a_{3}=(1,2,0), a_{4}=(0,3,1), \\
& a_{5}=(0.5,2.5,1), a_{6}=(1,0,0), a_{7}=(0,0,1), a_{8}=(0.5,0,1) .
\end{aligned}
$$

By Faísca et al. (2007), one can obtain:

$$
\begin{aligned}
I R & =\left\{\left(x, y_{1}, y_{2}\right): 0 \leq x \leq 0.5, y_{1}=3-x, y_{2}=1\right\} \\
& \cup\left\{\left(x, y_{1}, y_{2}\right): 0.5 \leq x \leq 1, y_{1}=3-x, y_{2}=2-2 x\right\} .
\end{aligned}
$$

The Figure 1, displays $S$ and $I R$. The $I R$ is denoted by the hatched lines.

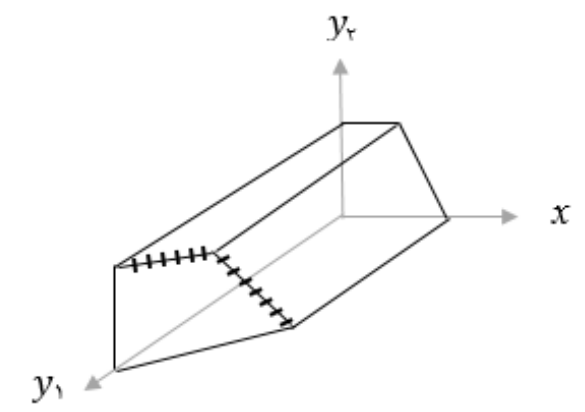

Figure 1: The constraint region $S$ and $I R$ of Example 5.1.

It is clear that $S$ and $I R$ are non-empty and $S$ is compact. Also, one can obtain:

$$
\begin{aligned}
P_{1}(x) & =\left\{\left(x, y_{1}, y_{2}\right) \in S: y_{1}=3-x, 0 \leq x \leq 3\right\}, \\
P_{2}(x)=\left\{\left(x, y_{1}, y_{2}\right) \in S: y_{2}=1,0 \leq x \leq 0.5\right\} & \cup\left\{\left(x, y_{1}, y_{2}\right) \in S: y_{2}=2-2 x, 0.5 \leq x \leq 1\right\} .
\end{aligned}
$$

Hence, $P_{i}(x) \neq \emptyset$, and $P_{i}(x)$ is a point-to-point map for all $x$, for $i=1,2$. Then, the Assumptions (1)-(3) hold, and we can apply the proposed algorithm. Using the proposed algorithm, the solution process is as follows:

Step 1. The MOLP $i$ problems, for $i=1,2$ are constructed as follows, respectively:

$$
\begin{aligned}
& \min \left\{\left(x, y_{2},-x-y_{2},-y_{1}\right):\left(x, y_{1}, y_{2}\right) \in S\right\}, \\
& \min \left\{\left(x, y_{1},-x-y_{1},-y_{2}\right):\left(x, y_{1}, y_{2}\right) \in S\right\} .
\end{aligned}
$$

In order to find the efficient sets $E_{i}, i=1,2$, using the described approach in Sayin (1996), one can obtain:

$$
E_{1}=\operatorname{conv}\left(a_{2}, a_{3}, a_{4}, a_{5}\right), E_{2}=\operatorname{conv}\left(a_{3}, a_{5}, a_{8}, a_{6}\right) \cup \operatorname{conv}\left(a_{5}, a_{4}, a_{7}, a_{8}\right) .
$$

The sets $E_{i}, i=1,2$ are drawn in Figure 2 (a) and (b) by the gray areas, respectively.

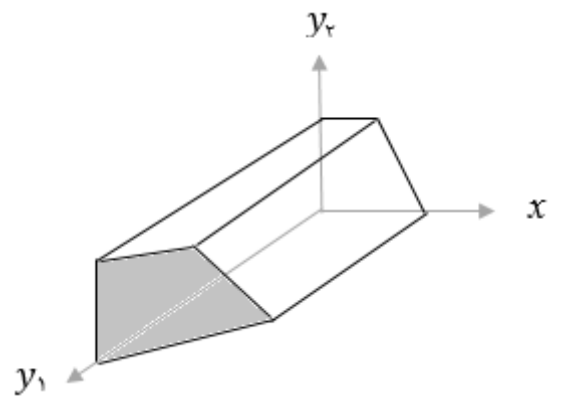

(a) $\mathrm{S}$ and $E_{1}$

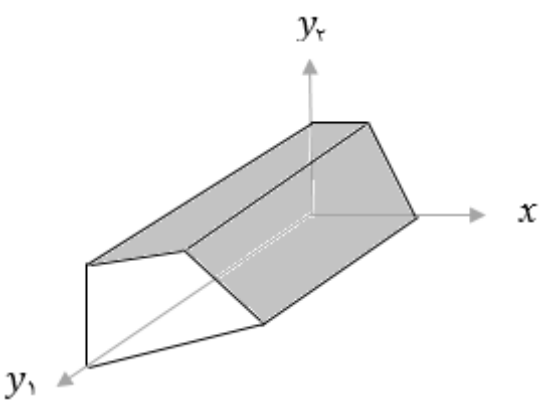

(b) $S$ and $E_{2}$

Figure 2: The constraint region $S, E_{1}$ and $E_{2}$ of Example 5.1. 
Step 2. Set $E=E_{1} \cap E_{2}$. So, we obtain:

$$
\begin{aligned}
& E=\left\{\left(x, y_{1}, y_{2}\right): 0 \leq x \leq 0.5, y_{1}=3-x, y_{2}=1\right\} \\
& \cup\left\{\left(x, y_{1}, y_{2}\right): 0.5 \leq x \leq 1, y_{1}=3-x, y_{2}=2-2 x\right\} .
\end{aligned}
$$

Here, it coincides with $I R$ which is shown in Figure 1 by the hatched lines.

Step 3. Solve the following LP:

$$
\min \left\{x+2 y_{1}-5 y_{2}:\left(x, y_{1}, y_{2}\right) \in S\right\} .
$$

The optimal solution is $X^{*}=(0,0,1)$ and $F\left(X^{*}\right)=-5$. Because $X^{*} \notin E$, go to Step 4 .

Step 4. Solve the following optimization problem:

$$
\min \left\{x+2 y_{1}-5 y_{2}:\left(x, y_{1}, y_{2}\right) \in E\right\} .
$$

Obviously, this problem is a non-convex optimization problem. According to the preceding discussions, we just consider the extreme points of $E$. We obtain $X^{*}=(0.5,2.5,1)$ as the optimal solution which is equal to the obtained optimal solution by the proposed approach in Faísca et al. (2007).

Example 5.2. Consider the following ULBMFP problem:

$$
\begin{gathered}
\min _{x} F\left(x, y_{1}, y_{2}\right)=-x+y_{1}-2 y_{2}, \\
\text { s.t. } x \geq 0 \\
\min _{y_{1}} f_{1}\left(x, y_{1}\right)=-y_{1}, \\
\text { s.t. } x+y_{1} \leqslant 8, \\
y_{1} \geq 0, x \geq 0 \\
\min _{y_{2}} f_{2}\left(x, y_{2}\right)=2 y_{2}, \\
\text { s.t. } x+y_{2} \leqslant 2 \\
y_{2} \geq 0, x \geq 0
\end{gathered}
$$

In this problem, we have:

$$
S=\left\{\left(x, y_{1}, y_{2}\right): x+y_{1} \leq 8, x+y_{2} \leqslant 2, x \geq 0, y_{1} \geq 0, y_{2} \geq 0\right\} .
$$

Also, the extreme points of $S$ are as follows:

$$
a_{1}=(0,0,0), a_{2}=(2,0,0), a_{3}=(2,6,0), a_{4}=(0,8,0), a_{5}=(0,8,2), a_{6}=(0,0,2) .
$$

By Faísca et al. (2007), one can obtain:

$$
I R=\left\{\left(x, y_{1}, y_{2}\right): 0 \leq x \leq 2, y_{1}=8-x, y_{2}=0\right\} .
$$

The Figure 3, displays $S$ and $I R$. The $I R$ is denoted by the hatched lines.

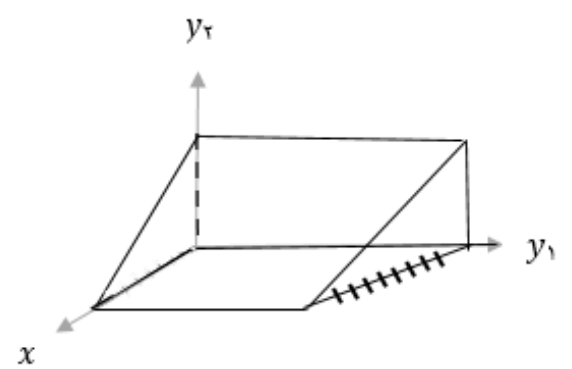

Figure 3: The constraint region $S$ and $I R$ of Example 5.2. 
Note that the sets $S$ and $I R$ are non-empty and $S$ is compact. Also, one can obtain:

$$
\begin{aligned}
& P_{1}(x)=\left\{\left(x, y_{1}, y_{2}\right) \in S: y_{1}=8-x, 0 \leq x \leq 8\right\} \\
& P_{2}(x)=\left\{\left(x, y_{1}, y_{2}\right) \in S: y_{2}=0,0 \leq x \leq 2\right\}
\end{aligned}
$$

Hence, $P_{i}(x) \neq \emptyset$, and it is a point-to-point map for all $x$, for $i=1,2$. Thus, the Assumptions (1)-(3) hold, and we can apply the proposed algorithm. The solution process is as follows:

Step 1. The MOLP $i$ problems, for $i=1,2$ are constructed as follows, respectively:

$$
\begin{aligned}
& \min \left\{\left(x, y_{2},-x-y_{2},-y_{1}\right):\left(x, y_{1}, y_{2}\right) \in S\right\} \\
& \min \left\{\left(x, y_{1},-x-y_{1}, 2 y_{2}\right):\left(x, y_{1}, y_{2}\right) \in S\right\}
\end{aligned}
$$

In order to find the sets $E_{i}, i=1,2$, using the described approached in Sayin (1996), one can obtain:

$$
E_{1}=\operatorname{conv}\left(a_{3}, a_{4}, a_{5}\right), E_{2}=\operatorname{conv}\left(a_{1}, a_{2}, a_{3}, a_{4}\right)
$$

The sets $E_{i}, i=1,2$ are drawn in Figure 4 (a) and (b) by the gray areas, respectively.

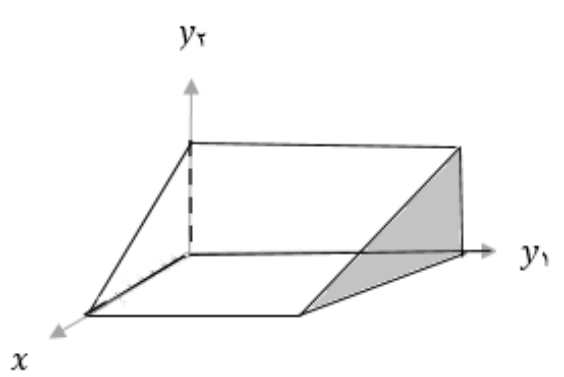

(a) $S$ and $E_{1}$

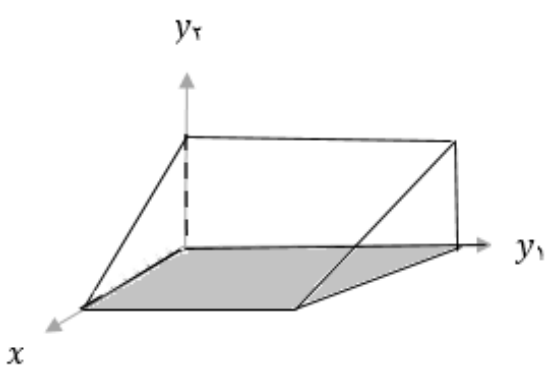

(b) $S$ and $E_{2}$

Figure 4: The constraint region $S, E_{1}$ and $E_{2}$ related to Example 5.2.

Step 2. Set $E=E_{1} \cap E_{2}$. We obtain $E=\gamma\left[a_{3}, a_{4}\right]=I R$. It is shown in Figure 3 by the hatched lines.

Step 3. Solve the following LP:

$$
\min \left\{-x+y_{1}-2 y_{2}:\left(x, y_{1}, y_{2}\right) \in S\right\} \text {. }
$$

The extreme optimal solution is $X^{*}=(0,0,2)$ and $F\left(X^{*}\right)=-4$. Because $X^{*} \notin E$, go to Step 4 .

Step 4. Solve the following optimization problem:

$$
\min \left\{-x+y_{1}-2 y_{2}:\left(x, y_{1}, y_{2}\right) \in E\right\} \text {. }
$$

Since $E$ is convex, this problem is a linear programming problem. We obtain $X^{*}=(2,6,0)$ as an optimal solution. It is the same as the obtained optimal solution by the proposed approach in Faísca et al. (2007).

\section{Conclusions}

In this study, we have investigated the relationship between the ULBMFP problems and the MOLP problems. We defined $k$ MOLP problems, and showed that the extreme points of the set of efficient solutions for all of these $k$ MOLP problems are identical to the extreme points of feasible solutions set for the ULBMFP problem with $k$ followers. Moreover, we studied the geometric properties of feasible solutions set of ULBMFP problem and we showed that for solving the ULBMFP problem, the leader objective function can be optimized over a certain efficient set. Furthermore, we presented an algorithm to solve the ULBMFP problems. Further studies can be concentrated on the implementation of the proposed algorithm upon large-scale ULBMFP problems. 


\section{Acknowledgements}

We are grateful to the Research Council of Shahid Chamran University of Ahvaz for the financial support under the grant number SCU.MM98.704

\section{References}

1. Alipour, M., Zare, K., and Seyedi, H. (2018). A multi follower bi-level stochastic programming approach for energy management of combined heat and power micro-grids. Energy, 149:135-146.

2. Bard, J. (1998). Practical Bilevel Optimization. Kluwer Academic, Dordrecht.

3. Bazaraa, M., Jarvis, J., and Sherali, H. (1977). Linear Programming and Network Flows. Wiley, New York.

4. Bazaraa, M., Sherali, H., and Shetty, C. (2006). Nonlinear Programming, Theory and Algorithms. Wiley, New York.

5. Benson, H. (1984). Optimization over the efficient set. Journal of Mathematical Analysis And Applications, 98:562-580.

6. Calvete, H. and Galé, C. (2007). Linear bilevel multi-follower programming with independent followers. Journal of Global Optimization, 39:409-417.

7. Dempe, S. (2003). Foundations of Bilevel Programming. Kluwer Academic, Dordrecht.

8. Ehrgott, M. (2005). Multicriteria Optimization. Springer-Verlag, Berlin, Germany.

9. Faísca, N., Saraiva, P., Rustem, B., and Pistikopoulos, E. (2007). A multi-parametric programming approach for multilevel hierarchical and decentralized optimization problems. Computer Management Sciences, 6:377397.

10. Fülöp, J. (1993). On the equivalency between a linear bilevel programming problem and linear optimization over the efficient set. Technical Report WP 93-1, Laboratory of Operations Research and Decision Systems, Computer and Automation Institute, Hungarian Academy of Sciences.

11. Glackin, J., Ecker, J., and Kupferschmid, M. (2009). Solving bilevel linear programs using multiple objective linear programming. Journal of Optimization Theory and Applications, 140(2):197-212.

12. Horst, R., Thoai, N., Yamamoto, Y., and Zenke, D. (2007). On optimization over the efficient set in linear multicriteria programming. Journal of Optimization Theory and Applications, 134:433-443.

13. Jorge, J. (2005). A bilinear algorithm for optimizing a linear function over the efficient set of a multiple objective linear programming problem. Journal of Global Optimization, 31:1-16.

14. Lu, J., Shi, C., Zhang, G., and Dillon, T. (2007). Model and extended kuhn-tuker approach for bilevel multifollower decision making in a refrential-uncoopeartive situation. Journal of Global Optimization, 38:597-608.

15. Ma, W., Wang, M., and Zhu, X. (2014). Improved particle swarm optimization based approach for bilevel programming problem-an application on supply chain model. International Journal of Machine Learning and Cyber, 5:281-292.

16. Metev, B. (2007). Multiobjective optimization methods help to minimize a function over the efficient set. Cybernetics and Information Technologies, 7:22-28.

17. Miao, C., Du, G., and Zandhang, R. J. T. (2017). Coordinated optimization of platform-driven product line planning by bilevel programming. International Journal of Production Research, 55:3808-3831.

18. Pieume, C., Fotso, L., and Siarry, P. (2008). An approach for finding efficient points in multiobjective linear programming. Journal of Information and Optimization Science, 29:203-216.

19. Pieume, C., Fotso, L., and Siarry, P. (2009). Solving bilevel programming problems with multicriteria optimization techniques. Opsearch, 42:169-183.

20. Sadeghi, H. and Moslemi, F. (2019). A multiple objective programming approach to linear bilevel multifollower programming. AIMS Mathematics, 4:763-778.

21. Safaei, A., Farsad, S., and Paydar, M. (2018). Robust bi-level optimization of relief logistics operation. Applied Mathematical Modelling, 56:359-380.

22. Sayin, S. (1996). An algorithm based on facial decomposition for finding the efficient set in multiple objective linear programming. Operations Research Letters, 19:87-94.

23. Steure, R. (1986). Multiple Criteria Optimization: Theory, Computation, and Application. Wiley, New York.

24. Wen, U. and Hsu, S. (1991). Efficient solutions for the linear bilevel programming problem. European Journal of Operational Research, 62:354-362.

25. Zhang, G., Lu, J., and Gao, Y. (2016). Multi-Level Decision Making: Models, Methods and Applications. 
Springer-Verlag Berlin, AN. 